\title{
AXIOMS FOR HOPF INVARIANTS
}

\author{
BY J. M. BOARDMAN AND B. STEER
}

Communicated by F. P. Peterson, July 19, 1966

1. Since $H$. Hopf first proved that $\pi_{3}\left(S^{2}\right) \approx \boldsymbol{Z}$ by showing that a certain invariant was nontrivial, several similar invariants-called generalized Hopf invariants-have been described; indeed, there are no less than 6 different definitions in the literature, and it was not always clear whether theorems about one applied to others. Many definitions were riddled with choices which had to be made, a notable exception being the definition of I. M. James [5]. This note gives an axiomatic approach somewhat similar to the axiomatic approach to Steenrod operations. Detailed proofs of the results below, together with some other properties of Hopf invariants, will appear elsewhere.

2. Denote by $\mathfrak{C}_{+}$the category whose objects are topological spaces with base-point and whose maps are homotopy classes of continuous base-point preserving maps between the spaces. $\delta_{+}$denotes the subcategory whose objects are countable connected CW-complexes with base-point a vertex; and $\mathcal{F}_{+}$denotes the subcategory whose objects are finite connected simplicial complexes with base-point a vertex. In both cases the maps are all the homotopy classes of continuous base-point preserving maps; and we shall suppose this is the case for any subcategory of $\mathrm{e}_{+}$considered. If $X$ is a space and $A$ a subspace $X / A$ denotes the identification space obtained by collapsing $A$ to a point and with base-point this point. The circle $S^{1}$ with base-point $(1,0)=e$ lies in $\mathcal{F}_{+}$. The functors

$$
E: \mathfrak{e}_{+} \rightarrow \mathfrak{e}_{+}, \vee: \mathfrak{e}_{+} \times \mathfrak{e}_{+} \rightarrow \mathfrak{e}_{+}, \wedge: \mathfrak{e}_{+} \times \mathfrak{e}_{+} \rightarrow \mathfrak{e}_{+}
$$

are defined by (i) $E A=A \times S^{1} / a_{0} \times S^{1} \cup A \times e$, (ii) $A \bigvee B=A \cup B / a_{0} \cup b_{0}$, (iii) $A \wedge B=A \times B / A \times b_{0} \cup a_{0} \times B$; where $a_{0}, b_{0}$ are the base-points of $A, B$ respectively. Given $X, A \in \mathbb{C}_{+}$we shall denote $\operatorname{Hom}_{\mathfrak{C}_{+}}(X, A)$, the homotopy classes of continuous base-point preserving maps of $X$ into $A$, by $[X, A]:$ and if this has the natural structure of a group we shall write + for the operation even when this operation may not be commutative. (Together with induced maps the operation $[\cdot, \cdot]$ defines a functor from $\mathfrak{e}_{+}^{*} \times \mathfrak{e}_{+}$to sets.) Moreover, if $X \in \mathfrak{e}_{+}$we can consider the cohomology theories $H_{A}^{i}(X)=\left[E^{i} X, A\right]$, where $i>0$ and $A \in \mathfrak{C}_{+}$. Then there are pairings between the theories $H_{A}^{*}(\cdot)$ and $H_{B}^{*}(\cdot)$ to the theory $H_{A \wedge B}^{*}(\cdot)$ defined as follows. Let $f \in \alpha \in H_{A}^{t}(X)$ $=\left[E^{i} X, A\right], g \in \beta \in H_{B}^{j}(X)=\left[E^{j} X, B\right]$. Then the homotopy class of 
the map $(f \wedge g) \bigcirc \Delta^{\prime}: E^{i+j} X \rightarrow A \wedge B$, where $\Delta^{\prime}: E^{i+j} X \rightarrow E^{i} X \wedge E^{j} X$ is defined by $\Delta^{\prime}\left(x, t_{1}, \cdots, t_{i+j}\right)=\left(x, t_{1}, \cdots, t_{i}, x, t_{i+1}, \cdots, t_{i+j}\right)$, is called the cup-product of $\alpha$ and $\beta$ and written $\alpha \cdot \beta$.

3. Definition. A Hopf $n$-ladder is a sequence of natural transformations $\lambda_{p}, 1 \leqq p \leqq n$, from the functor $[E \cdot E \cdot]$ to the functor $\left[E^{p} \cdot, \Lambda^{p}(E \cdot)\right]$ on a subcategory of $\mathfrak{C}_{+}^{*} \times \mathfrak{C}_{+}$containing $\mathcal{F}_{+}^{*} \times \mathfrak{S}_{+}$such that

(i) $\lambda_{1}=$ identity,

(ii) $\lambda_{p}(E \alpha)=0$ if $\alpha \in[X, A], n \geqq p>1$,

(iii) $\lambda_{p}(\alpha+\beta)=\lambda_{p}(\alpha)+\sum_{i+j=p} \lambda_{i}(\alpha) \cdot \lambda_{j}(\beta)+\lambda_{p}(\beta), n \geqq p \geqq 1$.

With this definition we have the following theorems on the category $\mathcal{F}_{+}^{*} \times \mathcal{S}_{+}$.

Theorem 1 (Existence). A Hopf $n$-ladder exists for every $n \geqq 1$.

ThEOREM 2 (UNIQUENESS). There is only one Hopf $n$-ladder for each $n \geqq 1$.

It is natural to ask how these "Hopf invariants" agree with those defined by others. The proofs of the two theorems make this clear for some of them. One may show fairly easily that the James-Hopf invariants [5] satisfy these conditions. This establishes Theorem 1. (The theorem may also be proved using another explicit operation, to be found in the paper giving more detailed proofs.) Let $\sigma_{n}$ be the word on two letters 1,2 defined inductively by $\sigma_{1}=2, \sigma_{n}=\left[\sigma_{n-1}, 1\right]$; $1<n<\infty$. And let $\iota_{\sigma_{n}}$ be the Whitehead product associated to the basic commutator $\sigma_{n}$, where we use the convention for Whitehead products suggested by H. Samelson and by M. G. Barratt (see page 129 of [2]), and where we suppose chosen some system of basic commutators containing the commutators $\sigma_{n}, n \geqq 1$. Let $h_{n}$ be the HiltonHopf invariant associated to $\iota_{\sigma_{n}}$ [4]. (These were singled out by W. D. Barcus and M. G. Barratt in [1].) Theorem 2 is then a consequence of the generalization of the following proposition to the case when $K \in F_{+}, X \in S_{+}$.

Proposition. If $K \in E F_{+}, X \in E S_{+}, \alpha \in[E K, E X]$, and if $\lambda_{p}$, $1 \leqq p \leqq n$, is a Hopf $n$-ladder, then $\lambda_{p}(\alpha)=E^{p-1} h_{p}(\alpha), 1 \leqq p \leqq n$.

The proof of the proposition follows the lines of proof of Proposition 3.2 of [3]. First we calculate $\lambda_{n}$ on Whitehead products and on composition elements by the axioms (i), (ii), and (iii). This enables us to calculate $\left(\iota_{2}+\iota_{1}\right) \bigcirc \alpha$ directly as a composition and indirectly using the Hilton-Milnor decomposition. $\left(\iota_{1}, \iota_{2}\right.$ are the classes of the standard injections of $E X$ into the first and second factors of $E X \vee E X$ respec- 
tively, and $\alpha \in[E K, E X]$.) Because $X$ and $K$ are supposed to be suspensions, the cup-products may be ignored and upon equating the two formulae obtained we find the proposition. In the general case when $X$ and $K$ are not suspensions, cup-products are not zero and the same method yields the general (more complicated) proposition.

4. A desuspended version of the axioms and theorems may be formulated using the products " $\mid$ " of [6]. The conditions are required to hold only modulo an "ideal" generated by Whitehead products, and uniqueness is thus modulo this "ideal" which is killed by suspension.

\section{REFERENCES}

1. W. D. Barcus and M. G. Barratt, On the homotopy classification of the extensions of a fixed map, Trans. Amer. Math. Soc. 88 (1958), 57-74.

2. M. G. Barratt, Spaces of finite characteristic, Quart. J. Math. Ser. (2) 11 (1960), 124-136.

3. A. Haefliger and B. Steer, Symmetry of linking coefficients, Comment. Math. Helv. 39 (1965), 259-270.

4. P. J. Hilton, On the homotopy groups of unions of spheres, J. London Math. Soc. 30 (1955), 154-172.

5. I. M. James, On the suspension triad, Ann. of Math. (2) 63 (1956), 191-247.

6. B. Steer, Extensions of mappings into H-spaces, Proc. London Math. Soc. (3) 13 (1963), 219-272.

The University of Warwick, Coventry and

Hertford COLlege, OXford 